\title{
The clinical outcomes of marginal donor hearts: a single center experience
}

\author{
Soo Yong Lee
}

Department of Internal Medicine-Cardiology, Pusan National University Yangsan Hospital, Yangsan, Korea

Background: The average donor heart utilization rate is known to be $31.4 \%$ in South Korea, although reported to improve to $42.9 \%$ in 2019 , still very low compared with other countries. More than $60 \%$ of donor hearts are discarded for the reason of their age or medical condition. However, were the donors truly marginal, and doomed to have poor outcomes? Herein, we suggest an answer to the questions with reporting outcomes of single heart transplant $(\mathrm{HTx})$ center, which actively utilizes the marginal donors.

Methods: Consecutive 66 HTx done from June 2014 to March 2021 in a single tertiary hospital were analyzed. The marginal donor (MD) was defined as follows: a donor age $>55$ years, left ventricular ejection fraction $<50 \%$ or significant structural heart disease, cold ischemic time $>240$ minutes, declined more than 5 times by the listed candidates as not suitable for transplant. Preoperative characteristics of recipients and donors and postoperative hemodynamic data, primary graft dysfunction (PGD), and the survival rate were analyzed.

Results: A total of 29 recipients out of 66 received an organ from MDs. A significant difference in preoperative ECMO status between MD and non-marginal donor (NMD) group (34.5\% vs. $67.6 \%$ ) was noted. There was no statistically significant difference in any-PGD (24.1\% vs. $16.2 \% ; \mathrm{P}=0.623)$. The cardiac index recorded immediate postoperative, 6 hours, 12 hours, 18 hours showed slightly lower in MD group, however, caught-up NMD group after 24 hours. Also, long-term results represented by 5-year survival showed no significant difference between the two groups (survival rates $86.2 \%$ vs. $75.3 \% ; \mathrm{P}=0.92$ ).

Conclusions: Appropriately selected donor hearts declined as 'marginal donor' hearts may increase organ utilization without an increase in post-transplant PGD and mortality.

Corresponding author: Soo Yong Lee

E-mail: shonge0906@gmail.com

(c) The Korean Society for Transplantation

This is an Open Access article distributed under the terms of the Creative Commons Attribution Non-Commercial License (http://creativecommons.org/licenses/by-nc/4.0/) which permits unrestricted non-commercial use, distribution, and reproduction in any medium, provided the original work is properly cited. 